\title{
Automated data collection services at ESRF Massif-1
}

\author{
Matthew Bowler ${ }^{a}$, and Didier Nurizzo ${ }^{b}$ \\ ${ }^{a}$ EMBL, 71 Avenue des Martyrs, Grenoble, France, ${ }^{b}$ ESRF, 71 Avenue des Martyrs, Grenoble, France, \\ AuthorEmail:nurizzo@esrf.fr
}

Since July 2014, the ESRF offers to the macromolecular crystallography community a wide range of automatic services, from crystal characterisation to structure determination, on the beamline MASSIF-1. The "hands-off" data collection process includes the automatic sample loading, optical and X-ray centering, crystal measurement, crystallographic characterization, data collection strategy and data collection processing. Users have the option of tailoring the different steps according to the knowledge of their crystals. Several type of data collection is available ranging from standard native data collection to pseudo-helical SAD data collection. The automatic procedure is tracked using our LIMS database, ISPyB and off-site users follow in real time through the dedicated ISPyB web interface and retrieve their data via the interface. 